\title{
Population pharmacokinetics of ceftazidime in burn patients
}

\author{
Eric Dailly, Michel Pannier', Pascale Jolliet \& Michel Bourin \\ Clinical Pharmacology Department, Hôtel Dieu Hospital, Nantes, France, and ${ }^{1}$ Burn Care Department, Hôtel Dieu Hospital, Nantes, France
}

\begin{abstract}
Aim The aim of this study was to characterize, via a population pharmacokinetic approach, the pharmacokinetics of ceftazidime in burn patients who were not in the acute post-injury phase.

Methods The development of the pharmacokinetic model was based on data from therapeutic drug monitoring (41 patients, 94 samples). The estimation of population pharmacokinetic parameters and the selection of covariates (age, gender, body weight, size of burn and creatinine plasma concentration) that could affect the pharmacokinetics were performed with a nonlinear mixed effect modelling method. Results No relationship between covariates and the pharmacokinetic parameters was established with the exception of an inverse-linear relationship between creatinine plasma concentration and ceftazidime total clearance. The total clearance of ceftazidime was $2.721 \mathrm{~h}^{-1}$ [coefficient variation $(\mathrm{CV})=56.3 \%$ ] and the distribution volume of the central compartment was $0.281 \mathrm{~kg}^{-1}(\mathrm{CV}=13.2 \%)$ The transfer rate constants $(\mathrm{k} 12, \mathrm{k} 21)$ between the central and peripheral compartments were $0.06718 \mathrm{~h}^{-1}(\mathrm{CV}$ $=87.2 \%)$ and $0.001823 \mathrm{~h}^{-1}(\mathrm{CV}=82.7 \%)$, respectively. From these parameters, the total ceftazidime volume of distribution $\left(10.641 \mathrm{~kg}^{-1}\right)$ was calculated.

Conclusion The population parameters were different from those obtained in a previous study performed in fewer patients and in the early period after burn injury. In our study, the lower ceftazidime clearance could be explained by the relative decrease in ceftazidime elimination in relation to the burn area, and the higher ceftazidime volume of distribution in the presence of interstitial oedema, which could act as a reservoir from which ceftazidime returns slowly to the circulation.
\end{abstract}

Keywords: burns, ceftazidime, population pharmacokinetics

\section{Introduction}

Ceftazidime is a third-generation cephalosporin with a broad spectrum, which is widely used to treat severe infections in burn patients. Like other $\beta$-lactam agents, the pharmacokinetics of ceftazidime are defined by high renal excretion and a volume of distribution similar to that of the extracellular space [1]. Consequently, the changes in renal function in the early period after burn injury and in the following hypermetabolic phase probably affect the pharmacokinetics of ceftazidime, requiring a change in the dosing regimen [2]. During the acute phase $(48 \mathrm{~h})$ of the burn injury, fluid loss from the vascular system occurs as a result of loss of capillary

Correspondence: Eric Dailly, Laboratoire de Pharmacologie clinique, Institut de Biologie, Centre Hospitalier Interuniversitaire, 9 Quai Moncousu, 44093 Nantes Cedex, France. Tel.: + 3324008 4095; fax:+ 3324008 3996;

E-mail: eric.dailly@chu-nantes.fr

Received 7 February 2003, accepted 6 June 2003. integrity. Renal blood flow also decreases, probably causing a transient impairment in renal drug clearance. During the hypermetabolic phase (after $48 \mathrm{~h}$ ), an increased cardiac output leads to an enhanced rate of glomerular filtration [3]. A previous study investigated the pharmacokinetics of ceftazidime in eight burn patients, mainly in the early period after injury, using a nonpopulation pharmacokinetic analysis [4]. Substantial differences in its pharmacokinetics were observed compared with other patients, and there was significant interindividual variation. The aim of the present study was to characterize the pharmacokinetics of ceftazidime in the hypermetabolic phase of burn injury and to compare the results with the work of Walstad et al. [4]. To be representative of the whole population of burn patients, which is characterized by its high variability, a population pharmacokinetic approach using data obtained for therapeutic drug monitoring was followed, allowing the inclusion of a reasonable large number of patients, and sparse sampling. 


\section{Subjects and methods}

Subjects and sampling

Forty-one patients hospitalized in the intensive burn care unit of Hôtel Dieu Hospital, Nantes, France) were studied. All were over 18 years old with severe injuries (second- and third-degree thermal injuries ranging from $8 \%$ to $80 \%$ of their total body surface area) (Table 1 ). Ceftazidime concentration data (provided by the therapeutic monitoring service) were obtained during the hypermetabolic phase (not less than $48 \mathrm{~h}$ after burn injury). Data were collected retrospectively over a 4year period. Informed consent and ethics committee approval were not required as therapeutic monitoring was part of the routine medical care of these patients. No additional patient information was needed for this study, and the concentration data are presented anonymously in accordance with ethical guidelines. The blood sampling was performed when the drug had reached steady state.

\section{Ceftazidime analysis}

A 1-ml aliquot of plasma was combined with $1 \mathrm{ml}$ acetonitrile, and the mixture was vortex-mixed and centrifuged at $1800 \mathrm{~g}$ for $5 \mathrm{~min}$ at $4{ }^{\circ} \mathrm{C}$. The supernatant $(1.6 \mathrm{ml})$ was added to dichloromethane $(8 \mathrm{ml})$, and the tubes were horizontally shaken for $10 \mathrm{~min}$ and centrifuged at $1800 \mathrm{~g}$ for $5 \mathrm{~min}$ at $4{ }^{\circ} \mathrm{C}$. Fifty microliters of the upper aqueous layer were injected into the high performance liquid chromatographic system. The mobile phase $\left(\mathrm{Na}_{2} \mathrm{HPO}_{4} 0.05 \mathrm{~mol} \mathrm{l}{ }^{-1}, \mathrm{pH} 2.5\right.$ /acetonitrile: 90/

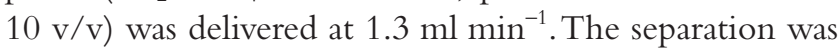
performed on a Nucleosil C18 column $(150 \times 4.6 \mathrm{~mm}$ ID; Colochrom, Gagny, France). Ceftazidime was detected by ultraviolet absorbance at $255 \mathrm{~nm}$. The limit of quantification was $0.5 \mathrm{mg} \mathrm{l}^{-1}$, the assay was linear up to $20 \mathrm{mg}^{-1}$. The degree of inaccuracy was $<3 \%$ ) and coefficients of variation $(\mathrm{CV})$ were $<6 \%$.

\section{Estimation of population parameters}

Structural models Data were analysed by a nonlinear mixed effect modelling method using MicroPharm population software (version 5.5; Institut National de la Science et de la Recherche Médicale). Two kinds of structural models (one-compartment and twocompartment) were tested. The parameters estimated in the final structural model were $C$ (total clearance of ceftazidime), V1 (apparent volume of the central compartment), k12 (transfer rate constant between the central and peripheral compartments) and k21 (transfer rate constant between the peripheral and central compartments).
Table 1 Characteristics of the burn patients studied and the number of blood samples taken.

Number (males)

$41(26)$

Age (year)

$46.2 \pm 18.5$

Body weight $(\mathrm{kg})$

Creatinine plasma concentration $(\mu \mathrm{mol} / \mathrm{l})$

$73.5 \pm 14.6$

Burned surface area (percent of the total body surface)

$75 \pm 56$

Total number of samples

$34 \pm 18$

Median number of samples per patient

94

2

The results are presented as mean \pm standard deviation.

Interindividual and residual variability models The interindividual and residual variabilities were modelled using the following equations.

$$
\theta_{\mathrm{i}}=\theta_{\mathrm{pop}}(1+\eta)
$$

where $\theta_{\mathrm{i}}$ is the individual estimate of pharmacokinetics parameter, $\theta_{\mathrm{pop}}$ is the population parameter estimate, and $\eta$ is the individual variation from the population parameter estimate.

$$
\mathrm{C}(\mathrm{t})_{\mathrm{i}, \mathrm{j}}=\mathrm{C}(\mathrm{t}) \operatorname{pred}_{\mathrm{i}, \mathrm{j}}(1+\Sigma)
$$

where $C(t)_{\mathrm{i}, \mathrm{j}}$ is the measured plasma concentration of ceftazidime for subject $\mathrm{i}$ at time $\mathrm{j}, C(t)$ pred $_{\mathrm{i}, \mathrm{j}}$ is the plasma predicted concentration of ceftazidime for subject $i$ at time $\mathrm{j}$, and $\Sigma$ is the residual error.

Selection of pharmacokinetics models In the first step of the analysis, the basic pharmacokinetic model without any covariates was defined [5]. The Akaike information criterion (Akaike value $=$ objective function $+2 \times \mathrm{P}$, where $\mathrm{P}$ is the number of estimated parameters and the objective function is similar to the function estimated by NONMEM) decreased from 722 for the onecompartment model to 712 for the two-compartment model, which indicated that the latter is better than the former model. The values for precision, which is the square root of the mean of square difference between $C_{\text {obs }}$ (observed plasma concentration of ceftazidime) and $C_{\text {pred }}$ (model predicted concentration of ceftazidime), were comparable (29.35 for the two-compartment model vs 30.86 for the one-compartment model). However, an improvement due to the two-compartment model appeared through the values of the bias, which is the mean difference between $C_{\text {obs }}$ and $C_{\text {pred }}(-6.497$ for the model with two compartments vs -9.113 for the model with one compartment). Overall, the two-compartment model best fitted the data.

In the second step, the influence of each patient covariate on $\mathrm{Cl}, \mathrm{V} 1, \mathrm{k} 21$ and $\mathrm{k} 12$ was tested. The covariates were age, gender, body weight, size of burn and creatinine plasma concentration. The full and reduced models were compared using the chi-squared test. A decrease of 
a least $3.6(P<0.05$, one degree of freedom $)$ of the objective function value compared to the basic pharmacokinetic model was required for the addition of a single parameter in the model. An intermediate multivariate model was obtained including all significant covariates. To keep only the covariates with the largest contribution in a final multivariate model, a change of 10.82 $(P<0.001)$ of the objective function in the final multivariate model was required for the retention of a single parameter during backward stepwise multiple regression analysis. Models were assessed by comparison of their respective objective function values and by visual examination of residual plots. The predictive performance of the model was assessed in terms of bias and precision.

\section{Results}

The plasma ceftazidime concentration data normalized for daily dose per kg bodyweight are shown in Figure 1. The population characteristics are presented in Table 1. Most of the patients did not suffer from renal failure, as the mean creatinine plasma concentration $\left(75 \mu \mathrm{mol} \mathrm{l^{-1 } )}\right.$ lies well within the normal range in male healthy volunteers $\left(60-120 \mu \mathrm{mol} \mathrm{l}^{-1}\right)$.

In the preliminary screening phase, the covariates that individually reduced the objective function by more than 3.6 units were the size of burn, age and creatinine plasma concentration. V1 was mainly influenced by the size of burn (linear relationship), age and creatinine plasma concentration (linear inverse relationship), $\mathrm{Cl}$ was influenced by creatinine plasma concentration (linear inverse relationship), k12 was influenced by age and creatinine plasma concentration (linear inverse relationship) and k21 was influenced by age and creatinine plasma concentration (linear relationship). In the forward multivariate

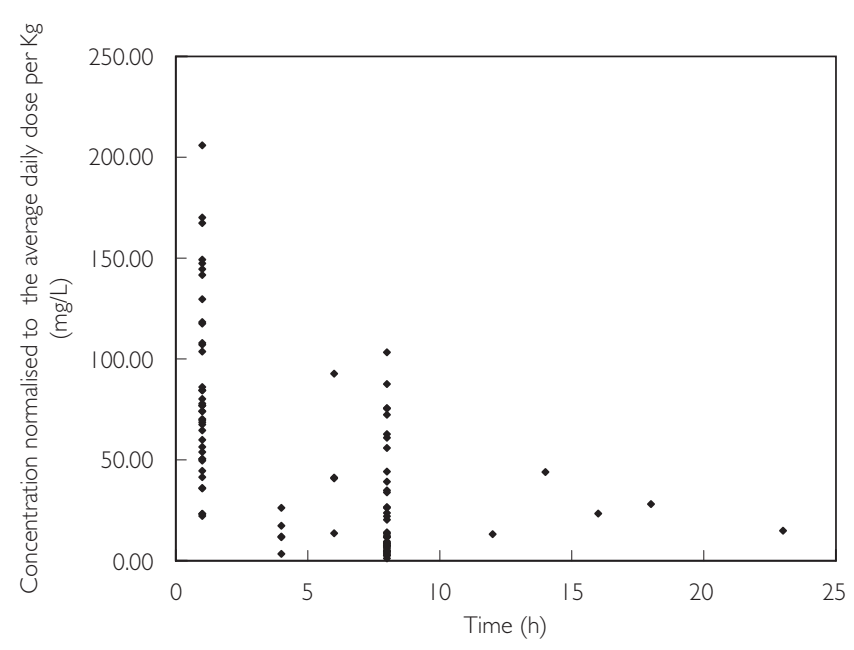

Figure 1 Ceftazidime plasma concentrations vs time in 41 burn patients. Concentrations were normalized to the mean daily dose per $\mathrm{kg}$. model-building, the size of burn, age and creatinine plasma concentration could be included cumulatively. In the backward elimination phase, only creatinine plasma concentration influenced ceftazidime clearance, exceeding the objective function cut-off value of 10.82 when it was omitted individually from the model. According to this result, the final model included a linear-inverse relationship between ceftazidime clearance and creatinine plasma concentration. With this model, the objective function decreased from 694 (two-compartment model without creatinine plasma concentration) to 647 (twocompartment model with creatinine plasma concentration). This improvement appears as a result of the relationship between $C_{\text {pred }}$ vs $C_{\text {obs }}$ and that between weighted residuals $\left(C_{\mathrm{obs}}-C_{\mathrm{pred}}\right) / C_{\text {pred }} v s C_{\text {pred }}$. The equation established by linear regression $\left[C_{\text {pred }}=24.9+0.60\right.$ $\times C_{\text {obs }}$ with a two-compartment model vs $C_{\text {pred }}=$ $14.0+0.76 \times C_{\text {obs }}$ with a two-compartment model integrating the creatinine plasma concentration] is closer to the theoretical equation $\left[C_{\text {pred }}=C_{\mathrm{obs}}\right]$ with the twocompartment model integrating the creatinine plasma concentration (Figures 2.1 and 2.2, Table 2). Identically, the equation established by linear regression $\left[\left(C_{\mathrm{obs}}-C_{\text {pred }}\right) /\right.$ $C_{\text {pred }}=0.154-0.0038 \times C_{\text {pred }}$ with a two-compartment model vs $\left(C_{\text {obs }}-C_{\text {pred }}\right) / C_{\text {pred }}=0.120-0.0024 \times C_{\text {pred }}$ with a two-compartment model integrating the creatinine plasma concentration] is closer to the theoretical equation $\left[\left(C_{\text {obs }}-C_{\text {pred }}\right) / C_{\text {pred }}=0\right]$ with the two-compartment model integrating the creatinine plasma concentration (Figures 3.1 and 3.2). Moreover, the precision (29.35 for the twocompartment model without creatinine plasma concentration vs 23.58 for the two-compartment model with creatinine plasma concentration) and the bias $(-6.497$ for the two-compartment model without creatinine plasma concentration $v s-3.20$ for the two-compartment model with creatinine plasma concentration) were improved. The convergence of these different elements showed that the best model was the two-compartment model integrating an inverse-linear relationship between ceftazidime clearance and creatinine plasma concentration.

With this model, the residual variability is $31.2 \%$ and the population values of pharmacokinetic parameters are presented in Table 3. The volume of ceftazidime of distribution at steady state $\left(10.641 \mathrm{~kg}^{-1}\right)$ was estimated from these parameters $\left(\mathrm{V} 1=0.281 \mathrm{~kg}^{-1}, \mathrm{Cl}=2.721 \mathrm{~h}^{-1}\right.$, $\left.\mathrm{k} 12=0.06718 \mathrm{~h}^{-1}, \mathrm{k} 21=0.001823 \mathrm{~h}^{-1}\right)$. The half-life values in the two-compartment model were calculated from the transfer rate constants $\mathrm{k} 12$ and $\mathrm{k} 21$ and are $10.3 \mathrm{~h}$ and $380.2 \mathrm{~h}$, respectively.

\section{Discussion}

Ceftazidime is widely used to treat burn patients, but few data are available on its pharmacokinetics in this group. 
Table 2 Observed concentrations $\left(C_{\mathrm{obs}}\right)$ vs predicted concentrations obtained by a mathematical model without creatinine plasma concentration $\left(C_{\text {pred }} 1\right)$ and predicted concentrations obtained by a mathematical model integrating creatinine plasma concentration $\left(C_{\text {pred }} 2\right)$.

\begin{tabular}{|c|c|c|c|c|c|c|c|}
\hline Patient & $C_{o b s}\left(m g l^{-1}\right)$ & $C_{\text {pred }} 1\left(m g l^{-1}\right)$ & $C_{\text {pred }} 2\left(m g l^{-1}\right)$ & Patient & $C_{o b s}\left(m g l^{-1}\right)$ & $C_{\text {pred }} 1\left(m g l^{-1}\right)$ & $C_{\text {pred }} 2\left(m g l^{-1}\right)$ \\
\hline \multirow[t]{4}{*}{1} & 1.8 & 11.79 & 1.836 & 22 & 7.3 & 23.59 & 63.24 \\
\hline & 22.8 & 51 & 39.93 & & 75.4 & 102 & 95.69 \\
\hline & 16.7 & 41.64 & 15.94 & 23 & 36 & 11.79 & 14.6 \\
\hline & 106.6 & 75.95 & 50.82 & & 95 & 51 & 55.97 \\
\hline \multirow[t]{2}{*}{2} & 47 & 27.57 & 67.09 & 24 & 63 & 23.59 & 71.41 \\
\hline & 109 & 43.31 & 68.05 & & 87 & 49.65 & 58.5 \\
\hline \multirow[t]{2}{*}{3} & 7.6 & 23.59 & 19.41 & 26 & 3 & 10.44 & 10.97 \\
\hline & 123 & 102 & 101.2 & & 34 & 49.65 & 52.11 \\
\hline \multirow[t]{3}{*}{4} & 14.3 & 23.59 & 13.66 & 27 & 12.8 & 45.62 & 34.07 \\
\hline & 82.5 & 102 & 94.53 & & 25.6 & 79.93 & 69.78 \\
\hline & 21.3 & 35.67 & 23.86 & 28 & 15 & 45.62 & 10.32 \\
\hline 6 & 79 & 102 & 92.13 & 30 & 17.7 & 13.78 & 12.08 \\
\hline \multirow[t]{2}{*}{7} & 14.6 & 23.59 & 18.47 & & 73 & 52.99 & 53.18 \\
\hline & 106 & 102 & 100.2 & 31 & 22.5 & 22.25 & 20.32 \\
\hline \multirow[t]{2}{*}{8} & 14.6 & 23.59 & 20.81 & & 66 & 100.7 & 102.3 \\
\hline & 106 & 102 & 102.8 & & 20.7 & 35.51 & 10.67 \\
\hline \multirow[t]{2}{*}{9} & 57.3 & 11.79 & 8.037 & & 78.5 & 113.9 & 90.16 \\
\hline & 109.5 & 51 & 48.7 & & 11.3 & 34.23 & 6.393 \\
\hline 10 & 20.5 & 23.59 & 11.24 & & 33.3 & 73.43 & 45.84 \\
\hline \multirow[t]{2}{*}{11} & 1.4 & 23.59 & 14.62 & 32 & 53 & 13.78 & 20.18 \\
\hline & 29 & 102 & 95.69 & & 100 & 52.99 & 61.8 \\
\hline 12 & 6.1 & 35.51 & 18.53 & 33 & 8.6 & 26.24 & 8.807 \\
\hline \multirow{3}{*}{16} & 67 & 52.99 & 47.73 & & 43.9 & 52.32 & 46.34 \\
\hline & 16.1 & 28.67 & 14.73 & 38 & 18.6 & 18.41 & 15.56 \\
\hline & 83.4 & 66.37 & 53.71 & & 82 & 57.62 & 56.8 \\
\hline \multirow[t]{2}{*}{17} & 8.3 & 23.59 & 11.72 & 39 & 10.2 & 22.25 & 22.12 \\
\hline & 79.2 & 102 & 92.13 & & 23.8 & 100.7 & 104.3 \\
\hline 18 & 5 & 27.57 & 11.01 & & 16 & 38.15 & 48.09 \\
\hline \multirow[t]{2}{*}{19} & 6.9 & 27.57 & 21.7 & & 140.5 & 116.5 & 131.3 \\
\hline & 52.9 & 106 & 103.6 & 40 & 8.3 & 28.89 & 10.73 \\
\hline \multirow[t]{2}{*}{20} & 30 & 23.59 & 40.15 & & 129.5 & 107.3 & 90.56 \\
\hline & 122 & 102 & 123.5 & 41 & 3.5 & 22.25 & 3.919 \\
\hline \multirow[t]{3}{*}{21} & 5.2 & 38.33 & 14.14 & & 66 & 100.7 & 80.5 \\
\hline & 55.4 & 72.64 & 48.87 & & & & \\
\hline & 22.8 & 59.98 & 21.6 & & & & \\
\hline
\end{tabular}

In a previous population study in burn patients [4], the volume of distribution at steady state was lower than in the present study $\left(0.381 \mathrm{~kg}^{-1}\right.$ vs $\left.10.61 \mathrm{~kg}^{-1}\right)$ and the total clearance was higher $\left(8.3 \mathrm{l} \mathrm{h}^{-1}\right.$ vs $\left.2.72 \mathrm{l} \mathrm{h}^{-1}\right)$. No correlation between the glomerular filtration rate estimated by creatinine clearance and the total clearance of ceftazidime was found, which is contrary to our results. Walstad et al. [4] explained this result by the loss of ceftazidime through the wound. This hypothesis is suggested by the increase of the nonrenal clearance, particularly in patients with a 

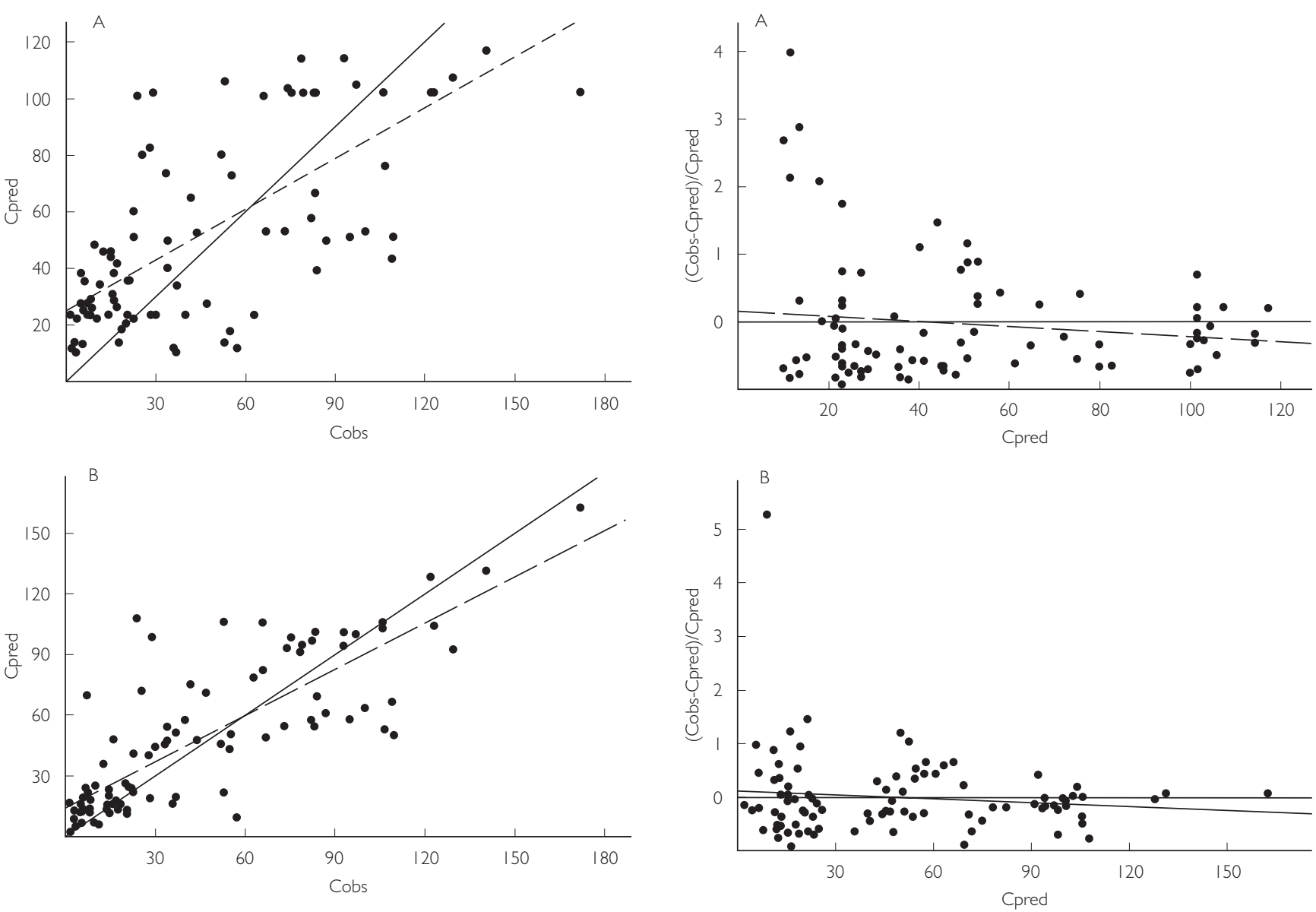

Figure 2 Relationship between $C_{\mathrm{obs}}$ and $C_{\mathrm{pred}} \cdot C_{\mathrm{obs}}$ are observed concentrations $\left(\mathrm{mg} \mathrm{l}^{-1}\right)$ and $C_{\text {pred }}$ are concentrations $\left(\mathrm{mg} \mathrm{l}^{-1}\right)$ predicted by the two-compartment model in Figure 2A and a twocompartment model integrating creatinine plasma concentration in Figure 2B. The straight-line equation (- - ) obtained by linear regression is $C_{\text {pred }}=24.9+0.60 \times C_{\text {obs }}$ in Figure $2 \mathrm{~A}$ and $C_{\text {pred }}=$ $14+0.76 \times C_{\text {obs }}$ in Figure $2 \mathrm{~B}$.

high burn surface area. To explore this additional elimination pathway, we tested unsuccessfully a potential linear relationship between ceftazidime clearance and the size of the burn area. However, the influence of the depth of burn injury on ceftazidime pharmacokinetics was not investigated because this parameter was not available in this retrospective study.

The average time from burn injury to administration of ceftazidime was 3.5 days (excluding one patient who received the drug 21 days after injury) in the study of Walstad et al. (the acute phase) vs 31.7 days in our study (the hypermetabolic phase). In spite of the higher renal elimination usually observed during the hypermetabolic phase compared with the acute phase, total ceftazidime clearance was lower in our study. This could be explained by a relative decrease in the nonrenal clearance, through the burn surface observed in the early period after burn injury. Indeed, no relationship between

Figure 3 Relationship between $\left(C_{\mathrm{obs}}-C_{\mathrm{pred}}\right) / C_{\text {pred }}$ and $C_{\text {pred. }} C_{\mathrm{obs}}$ are observed concentrations $\left(\mathrm{mg} \mathrm{l}^{-1}\right)$ and $C_{\text {pred }}$ are concentrations $\left(\mathrm{mg} \mathrm{l}^{-1}\right)$ predicted by the two-compartment model in Figure 3A and a two-compartment model integrating creatinine plasma concentration in Figure 3B. The equation obtained by linear regression is $\left(C_{\text {obs }}-C_{\text {pred }}\right) / C_{\text {pred }}=0.154-0.0038 \times C_{\text {pred }}$ in Figure $3 \mathrm{~A}$ and $\left(C_{\text {obs }}-C_{\text {pred }}\right) / C_{\text {pred }}=0.120-0.0024 \times C_{\text {obs }}$ in Figure $3 \mathrm{~B}$.

Table 3 Population pharmacokinetic parameters for ceftazidime obtained by nonlinear mixed effect modelling.

\begin{tabular}{llcc}
\hline & $\begin{array}{c}\text { Population } \\
\text { estimates }\end{array}$ & $\begin{array}{c}\text { SE of } \\
\text { estimates }\end{array}$ & $\begin{array}{c}\text { Interindividual } \\
\text { variability } \\
\text { CV percentage }\end{array}$ \\
\hline $\begin{array}{l}\text { Clearance }(\mathrm{L} / \mathrm{h}) \\
\text { Distribution volume of } \\
\quad \text { central compartment }(\mathrm{L} / \mathrm{kg})\end{array}$ & 0.72 & 0.088 & 56.3 \\
$\mathrm{k} 12\left(\mathrm{~h}^{-1}\right)$ & 0.06718 & 0.021 & 13.2 \\
$\mathrm{k} 21\left(\mathrm{~h}^{-1}\right)$ & 0.001823 & 0.1362 & 87.2 \\
\hline
\end{tabular}

the size of burn and the ceftazidime clearance was found. Consequently, the relationship between ceftazidime clearance and the glomerular filtration rate could have been restored. This relationship appears in our results through the influence of creatinine plasma con- 
centration as a covariate of ceftazidime clearance. The elevated volume of distribution observed in our study could be explained by an increase in the extracellular fluid volume. To compensate for the hypovolemia observed during the acute phase of the injury, large quantities of fluid are exogenously administered, expanding extracellular fluid volume and resulting in interstitial oedema. The ability of ceftazidime to substantially penetrate blister fluid was demonstrated by Zong et al. in the early post-burn stage [6]. Simultaneously, these authors observed that the half-life of elimination was prolonged although the nonrenal clearance of ceftazidime was increased. These results are consistent with the hypothesis that ceftazidime could diffuse into the oedematous fluid, which could act as a reservoir from which ceftazidime returns slowly to the circulation. Such a phenomenon has been described for ceftazidime in patients with ascites [7]. The two-compartment model is compatible with this hypothesis. The peripheral compartment could be the oedematous fluid reservoir, and the large difference in the values of the transfer rate constants between the central and the peripheral compartment could reflect the slow return of ceftazidime from the interstitial oedema to the central compartment.

The differences between our results and those of the previous study of Walstad et al. [4] could be explained by the pathological changes between the acute phase of burn injury and the hypermetabolic phase. This suggests that the intraindividual variability in ceftazidime pharmacokinetics can vary between the different phases after burn injury. Moreover, the interindividual variability in pharmacokinetics parameters is significant, which supports the validity of therapeutic monitoring of ceftazidime in burn patients. The population-based approach allowed the identification of a covariate that affects the pharmacokinetics of ceftazidime in burn patients. However, the residual variability was substantial, indicating the limitation of a retrospective study of the lower quality of the data compared with a prospective study [8]. A prospective population pharmacokinetic study would allow improvement of the model and the investigation of other covariates such as the depth of burn injury.

\section{References}

1 Rains CP, Bryson HM, Peters DH. Ceftazidime - An update of its antibacterial activity, pharmacokinetic properties and therapeutic efficacy. Drugs 1995; 49: 577-617.

2 Weinbren MJ. Pharmacokinetics of antibiotics in burn patients. J Antimicrob Chemother 1999; 44: 319-377.

3 Jaehde U, Sörgel F. Clinical pharmacokinetics in patients with burns. Clin Pharmacokinet 1995; 29: 15-28.

4 Walstad RA, Aanderud L, Thurmann-Nielsen E. Pharmacokinetics and tissue concentrations of ceftazidime in burn patients. Eur J Clin Pharmacol 1988; 35: 543-549.

5 Mouly S, Aymard G, Tillement JP, Caulin C, Bergmann JF, Urien S. Increased oral ganciclovir bioavailability in HIVinfected patients with chronic diarrhoea and wasting syndrome - a population pharmacokinetic study. Br J Clin Pharmacol 2001; 51: 557-565.

6 Zong G, Xia G, Zhang Y. The pharmacokinetics of ceftazidime in the burned patients. Zhonghua Zheng Xing Shao Shang Wai Ke Za Zhi 1994; 10: 385-388.

7 El Touny M, El Guinaidy MA, Abd El Barry M, Osman L, Sabbour MS. Pharmacokinetics of ceftazidime in patients with liver cirrhosis and ascites. J Antimicrob Chemother 1991; 28: 95100.

8 Antal EJ, Grasela TH Jr, Smith RB. An evaluation of population pharmacokinetics in therapeutic trials. Part III. Prospective data collection versus retrospective data assembly. Clin Pharmacol Ther 1989; 46: 552-559. 